\title{
Implementierung eines selbstfahrenden und individuell abrufbaren Personentransportsystems
}

\author{
Sven A. Beiker
}

\section{Inhaltsverzeichnis}

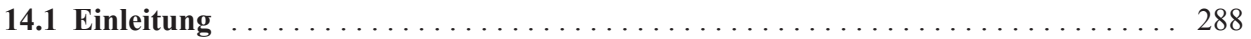

14.2 Begriffsbestimmung und Abgrenzung $\ldots \ldots \ldots \ldots \ldots \ldots \ldots \ldots \ldots \ldots \ldots \ldots \ldots \ldots \ldots \ldots \ldots \ldots$

14.3 Das selbstfahrende und individuell abrufbare Personentransportsystem . . . . . . 289

14.3.1 Technische Ausführung . . . . . . . . . . . . . . . . . . . . . . . . . . . . . . . . . 290

14.3.2 Operativer Betrieb . . . . . . . . . . . . . . . . . . . . . . . . . . . . . . . . . . . 292

14.3.3 Geschäftsmodell . . . . . . . . . . . . . . . . . . . . . . . . . . . . . . . . . 294

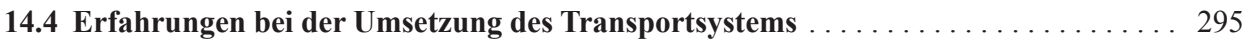

14.4.1 Evaluations-, Versuchs- und Öffentlichkeitsbetrieb . . . . . . . . . . . . . . . 295

14.4.2 Auswahl des Fahrzeugkonzepts . . . . . . . . . . . . . . . . . . . . . . 297

14.4.3 Risikobewertung und Rechtseinordnung . . . . . . . . . . . . . . . . . . 297

14.4.4 Vertragsgestaltung . . . . . . . . . . . . . . . . . . . . . . . . . . . . . . . 298

14.4.5 Auswahl des Betriebsgebiets und der Betriebsszenarien . . . . . . . . . . . . . 299

14.4.6 Einrichtung des Transportsystems und Zertifizierung des Personals . . . . . . . 301

14.4.7 Systemstart und Betriebsüberwachung . . . . . . . . . . . . . . . . . . . 302

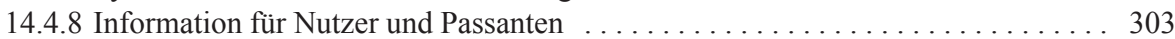

14.4.9 Öffentliche Reaktionen . . . . . . . . . . . . . . . . . . . . . . . . 304

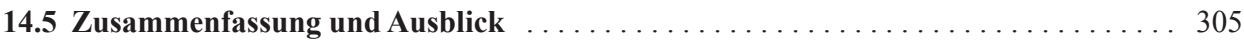

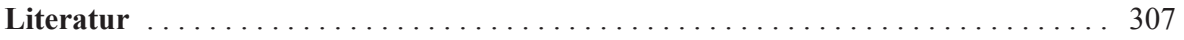

S. A. Beiker $(\bowtie)$

formerly Stanford University, Center for Automotive Research at Stanford, USA sven@svenbeiker.com 


\subsection{Einleitung}

Derzeit wird in der Fachwelt und in der Öffentlichkeit viel über automatisierte (oft auch ,autonom“ genannte) Fahrzeuge diskutiert und geforscht bzw. werden deren erste Produktumsetzungen entwickelt. Dabei steht häufig der individuell genutzte Pkw im Mittelpunkt der Betrachtung, d.h., der Blick richtet sich auf die zunehmende Automatisierung der Fahrzeuge auf Stadt- und Fernstraßen. Während die Vision, dass Pkw auf öffentlichen Straßen ohne menschlichen Eingriff selbsttätig fahren, noch in ferner Zukunft zu liegen scheint, gibt es bereits Beispiele, wo schon heute oder zumindest sehr bald selbstfahrende Fahrzeuge für den öffentlichen Personentransport eingesetzt werden. Nachdem schienengebundene Systeme über die letzten Jahrzehnte bereits erfolgreich ohne fahrzeugseitiges Personal betrieben wurden, wird nun auf der Straße die Schiene als „Leitmedium“ durch Satellitennavigation sowie Hinderniserkennung ersetzt, um dann eine automatisierte Fahrt zum Wunschziel des Nutzers zu ermöglichen.

Von solchen selbstfahrenden Transportsystemen kann bereits heute viel an Erfahrung für die zukünftige Einführung hochgradig automatisierter Pkw abgeleitet werden, obwohl diese Systeme häufig nur in einem begrenzten Gebiet wie dem Innenstadtbereich betrieben werden. So sind beispielsweise die betriebsbedingten, nutzerspezifischen, versicherungstechnischen oder haftungsrechtlichen Belange für langsam fahrende und gebietsbegrenzte Fahrzeuge im Kern oftmals ähnlich denen für Fahrzeuge auf Autobahnen. Damit steht zu hoffen, dass die hier beschriebenen selbstfahrenden und individuell abrufbaren Personentransportsysteme trotz unterschiedlicher Zielrichtungen im Vergleich zu hochgradig automatisierten Reiselimousinen Synergien aufzeigen, die für die Einführung letzterer in den öffentlichen Straßenverkehr wegweisend sein können. In diesem Beitrag wird von der Implementierung eines solchen Transportsystems an der Stanford Universität in Kalifornien berichtet. Anliegen dieses Berichts ist es, die hier gemachten Erfahrungen der zukünftigen Einführung anderer automatisierter Fahrzeuge zugutekommen zu lassen.

\subsection{Begriffsbestimmung und Abgrenzung}

In diesem Beitrag wird hauptsächlich von ,selbstfahrenden und individuell abrufbaren Personentransportsystemen“ bzw. derartigen Fahrzeugen gesprochen. Der Begriff ,,selbstfahrend" ist dabei in den Bereich der Fahrzeugautomatisierung einzuordnen: In Straßenfahrzeugen werden Computersysteme so eingesetzt, dass dem Menschen die Fahraufgabe abgenommen wird. In dem hier betrachteten Fall wird die Fahraufgabe entsprechend der „Navigation“, „Bahnführung“ und „Stabilisierung“ vollständig durch Computersysteme ausgeführt, und ein Fahrer ist damit nicht mehr erforderlich. Unter Umständen ist sogar keine einzige Person im Fahrzeug anwesend, wenn das Fahrzeug beispielsweise für logistische Zwecke eine Leerfahrt unternimmt. Diese Automatisierungsebene wird auch als „Vollautomatisierung“ entsprechend der Definition SAE J3016 [1] bezeichnet. 
Der Begriff ,individuell abrufbar“ (englischsprachig on-demand) beschreibt, dass das Transportsystem vom Nutzer für die individuelle Verwendung abgerufen werden kann (s. Kap. 2). Das kann beispielsweise über eine Smartphone-App erfolgen, d. h., der Nutzer kann ein Fahrzeug zum derzeitigen Standort anfordern, genauso wie ein Taxi, nur mit dem Unterschied, dass die Fahrzeuge des hier betrachteten Transportsystems keine Fahrer benötigen. Damit ergibt sich auch, dass der Begriff, Transportsystem“ als ein Gesamtkonzept aus verschiedenen Fahrzeugen sowie einer zentralen Infrastruktur, die die Fahrzeuge übergeordnet für den Nutzereinsatz koordiniert, verstanden wird.

Die Betrachtungen, die im vorliegenden Beitrag für ein solches Personentransportsystem angestellt werden, beziehen sich auf ein Ballungsgebiet, also einen bebauten Raum mit einer erhöhten Bevölkerungsdichte. Das bedeutet, dass eine urbane Bebauung mit Straßen, Parkplätzen, Radwegen, Fußwegen, Fußgängerzonen sowie Gebäuden angenommen wird, auf und zwischen denen das Transportsystem für die Personenbeförderung eingesetzt wird. Die Größe des Ballungsgebiets ist dabei weniger entscheidend als deren städtebaulicher Charakter, d.h., es ist unerheblich, ob es sich um eine sogenannte „MegaCity“ handelt oder lediglich um den Innenstadtbereich einer Mittel- oder gar Kleinstadt. Die hier betrachteten Personentransportsysteme werden für den generellen Einsatz dort betrachtet, wo der öffentliche Personentransport oder das privatgenutzte Automobil keine optimale Lösung für die Mobilitätsbedürfnisse von Nutzern darstellt.

\subsection{Das selbstfahrende und individuell abrufbare Personentransportsystem}

In diesem Beitrag wird ein konkretes Beispiel eines selbstfahrenden und individuell abrufbaren Personentransportsystems beschrieben, und zwar das Fahrzeug „Navia“ der französischen Firma Induct [2]. Ein solches Fahrzeug stand von Juli 2013 bis Februar 2014 an der Stanford Universität für Evaluationszwecke zur Verfügung, sodass der Einsatz dieses Fahrzeugs seit Anfang 2013 operativ gestaltet und dokumentiert werden konnte. Auf diesen empirischen Erfahrungen beruhen die nachfolgenden Ausführungen. Grundsätzlich ist festzuhalten, dass Fahrzeuge wie der Navia ein gutes Beispiel für einen allgemeinen Trend solcher Systeme sind [3, 4, 5, 6]. In diesem Zusammenhang sei ausdrücklich darauf hingewiesen, dass die spezielle Darstellung des Navia in keinster Weise eine Präferenz oder Bewertung des Systems im Vergleich zu anderen darstellt, auch hat die Firma Induct keinerlei Einfluss auf die Beschreibung der hier diskutierten Sachverhalte ausgeübt. Das bedeutet, dass die folgenden Beschreibungen zwar auf Grundlage der Erfahrungen mit dem Induct Navia an der Stanford Universität entstanden sind, im wissenschaftlichen und verkehrsplanerischen Sinne aber eine generelle Anwendbarkeit für selbstfahrende und individuell abrufbare Transportsysteme im Allgemeinen haben und bei der Umsetzung anderer Systeme grundsätzlich genutzt werden können. 


\subsubsection{Technische Ausführung}

Das für die Ausführungen in diesem Beitrag betrachtete selbstfahrende und individuell abrufbare Personentransportsystem nutzt Fahrzeuge, die mit Satellitennavigation, Laser, Kameras, Ultraschall, Lenkwinkel- und Raddrehwinkelsensoren ausgestattet sind. Mit diesen Sensoren und Systemen können Position und Umfeld des Fahrzeugs bestimmt und überwacht werden. Während die Satellitennavigation bereits eine recht genaue Positionsbestimmung ermöglicht (im Falle der Verwendung von zusätzlichen Korrekturmethoden bis in den Zentimeterbereich hinein), nutzen die hier betrachteten Systeme ein Verfahren, das als Simultaneous Localization and Mapping (SLAM) bezeichnet wird.

Für dieses Verfahren wird das Fahrzeug zunächst durch Betriebspersonal in dem geplanten Betriebsgebiet manuell gesteuert, während die Koordinaten der Satellitennavigation und die Daten der Laser-, Kamera-, und gegebenenfalls Ultraschallsysteme aufgezeichnet werden. Aus diesen Daten wird eine digitale Karte des Betriebsgebiets erstellt, die allerdings - anders als herkömmliche Karten - eine dreidimensionale Repräsentation des Gebiets ergibt. Diese Repräsentation beschreibt die stationäre Situation des Gebiets, d.h., alle Veränderungen, die im späteren Betrieb der Fahrzeuge gegenüber diesen gespeicherten Daten wahrgenommen werden, werden als bewegliche oder zumindest neu hinzugekommene Hindernisse eingeordnet. Diese Veränderungen sind damit besonders zu beachten und erfordern möglicherweise eine Abweichung von der vorprogrammierten Bahn.

Die SLAM-Technologie stellt damit eine „virtuelle Schiene“ dar, d. h., was für automatisierte Schienensysteme die physikalische Spurführung, ist für die hier betrachteten Systeme die Satellitennavigation als Referenzsystem in Verbindung mit der Umfelderfassung. Abweichungen zwischen der gespeicherten Repräsentation und der kontinuierlichen Umfelderfassung werden als Hindernisse eingeordnet, die gegebenenfalls eine Änderung der Routen- bzw. Bahnführung erfordern. Dabei dienen die Lasersensoren vorrangig zum Erfassen von Objekten (beispielsweise Personen, Fahrzeuge, Bebauung, Hindernisse) im Mittel- und Fernbereich mit einer Entfernung vom Fahrzeug von mehr als ca. einem Meter bis hin zu 200 Metern. Die Ultraschallsensoren werden für Objekterkennung im Nahbereich eingesetzt, also weniger als zwei Meter vom Fahrzeug entfernt. Die Kamerasysteme bieten darüber hinaus zusätzliche Informationen über die Kontur und damit Art des erfassten Objekts (z. B. Person oder Pflanze), sodass ein möglichst vollständiges Bild nach Art, Entfernung, Richtung und gegebenenfalls Geschwindigkeit der umgebenden Objekte entsteht.

Änderungen des Referenzsystems, also Hindernisse in der geplanten Route, werden in der zentralen Steuereinheit des Fahrzeugs berücksichtigt, sodass die tatsächliche Route fortlaufend unter Beachtung verschiedener Bewertungskriterien bestimmt werden kann. Somit werden je nach eingegebenem Fahrziel und augenblicklicher Verkehrs- und Umfeldsituation eine optimale Route und Bahn berechnet und ausgeführt. Selbst wenn augenblicklich keine Route befahren werden kann, beispielsweise weil die fahrzeugseitige Sensorik bei Annäherung an ein Hindernis erkennt, dass dieses nicht umfahren werden kann und auch keine Alternativroute verfügbar ist, würde das Fahrzeug dann die Fahrt bis zur Beseitigung des Hindernisses unterbrechen. 


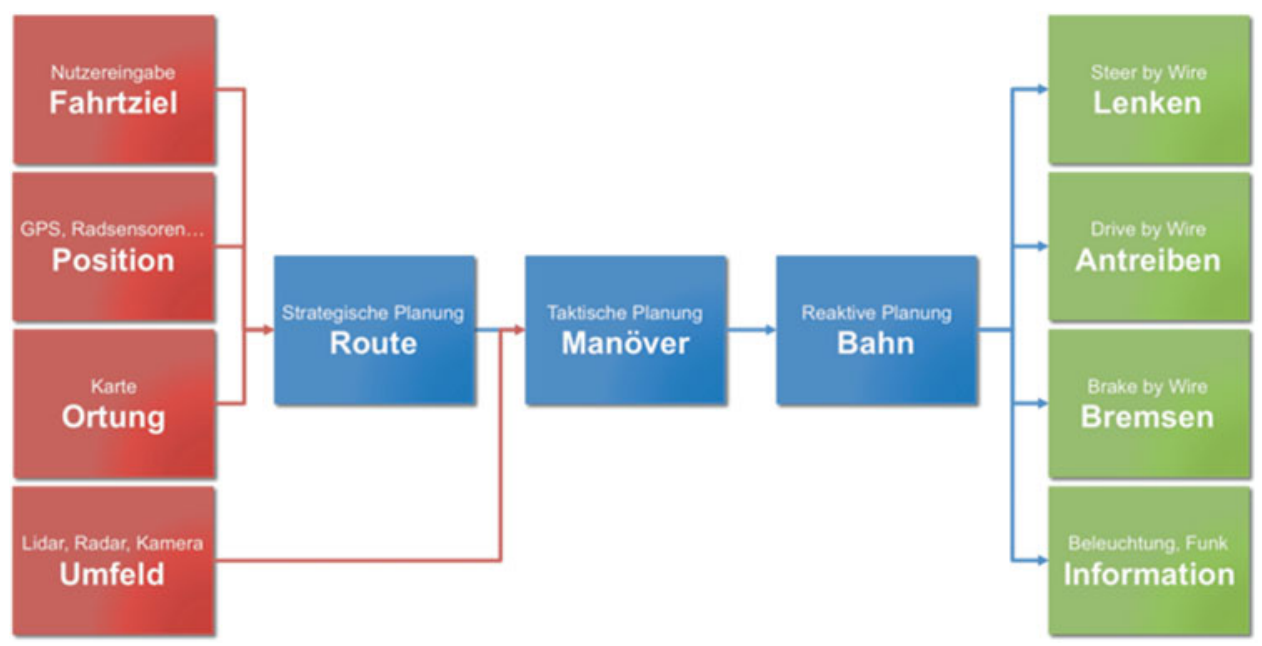

Abb. 14.1 Blockschaltbild eines selbstfahrenden Fahrzeugs (schematisch, vereinfacht)

Die Fahrbefehle werden an die elektrifizierten Lenkungs-, Brems- und Antriebssysteme zur Umsetzung übermittelt. Das bedeutet, das Fahrzeug verfügt über elektronische Schnittstellen für diese Komponenten, sodass die zentrale Steuereinheit des Fahrzeugs Lenk-, Brems- und Antriebsbefehle übermitteln kann und das Fahrzeug somit die Fahrt durch das Zusammenspiel von Umfelderkennung, Informationsverarbeitung und Fahrtwunsch selbstständig ausführt (s. Abb. 14.1). Dabei wird jede Achse einzeln über je einen bürstenlosen 48V/8kW-Elektromotor angetrieben. Das Fahrzeug verfügt über unabhängige Lenkungen an Vorder- und Hinterachse. Die technische Höchstgeschwindigkeit beträgt $40 \mathrm{~km} / \mathrm{h}$, ist für erste Anwendungen auf $20 \mathrm{~km} / \mathrm{h}$ limitiert und kann bei Bedarf weiter per Parametrierung begrenzt werden, der Wendekreisdurchmesser beträgt 3,5 Meter.

Der Benutzer des Fahrzeugs hat mehrere Eingriffsmöglichkeiten in den Fahrzeugbetrieb. Zum einen kann das Fahrzeug über einen Eingabebildschirm an einer festen Haltestelle abgerufen werden, im allgemeinen Fall wird das aber über eine Smartphone-App erfolgen. Wenn das Fahrzeug dann selbsttätig zu der angeforderten Einsteigeposition kommt, werden nach Fahrzeugstillstand die Parkbremsen angezogen und die Tür (in diesem Falle eine offene Stahlrohrkonstruktion) geöffnet, sodass Passagiere sicher in das Fahrzeug einsteigen können. Dann gibt der Benutzer über einen im Fahrzeug verbauten Eingabebildschirm das gewünschte Fahrziel ein, worauf sich die Tür wieder schließt und die Parkbremse gelöst wird, sodass sich das Fahrzeug entsprechend der nun geplanten Route in Bewegung setzen kann. Dabei haben Passagiere die Möglichkeit, das Fahrzeug jederzeit durch einen Notfallschalter sofort zu stoppen. In solch einem Fall können Passagiere auch über eine Kommunikationsanlage mit dem Betriebspersonal in Verbindung treten, um Hilfe anzufordern oder sonstige Belange zu klären. Da die Verbindung über Funkkommunikation erfolgt, kann das Betriebspersonal vollkommen unabhängig vom eigentlichen Betriebsgebiet der Fahrzeuge stationiert sein. 


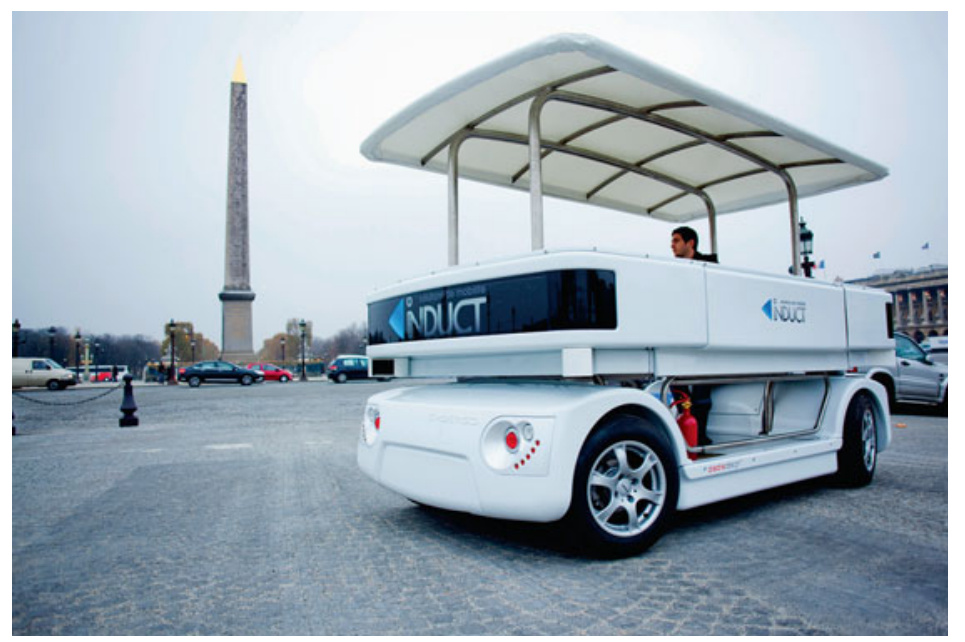

Abb. 14.2 Offene Bauweise des Induct Navia [2] (Abbildung mit freundlicher Genehmigung der Fa. Induct)

Wie Abb. 14.2 zeigt, zeichnet sich das hier betrachtete Fahrzeug durch eine sehr offene Bauweise aus. Oberhalb Hüfthöhe der Passagiere gibt es keine geschlossene Beplankung, sondern nur vier Stützen für das Baldachindach. Sitze sind in dem Fahrzeug ebenfalls nicht vorhanden, sondern nur Möglichkeiten für Passagiere, sich an gepolsterten Stützen anzulehnen. Das Fahrzeug bietet Platz für bis zu acht Personen (maximale Zuladung 800 Kilogramm); die Hauptabmessungen betragen 3,5 × 2,0 × 2,5 Meter (Länge $\times$ Breite $\times$ Höhe).

Die Stromversorgung der Fahrzeuge hat eine geschätzte Betriebsdauer von ca. acht Stunden über den chemischen Energiespeicher (Batterie) oder 20 Minuten über den kapazitiven Energiespeicher (Supercapacitor). Das Laden kann wahlweise außerhalb der Betriebszeiten über ein Kabel erfolgen oder während kurzer Betriebspausen über eine drahtlose Ladestation. Gerade die drahtlose Ladevariante ist für das fahrerlose Betriebsmodell von Vorteil, da die Fahrzeuge bei Bedarf selbstständig zur Ladestation fahren können und kein Personal erforderlich ist, um beispielsweise ein Ladekabel anzuschließen. Für den Betrieb an der Stanford Universität wird allerdings vorerst die Ladevariante per Kabel außerhalb der Betriebszeiten vorgezogen, da sich das für den zunächst geplanten Versuchsbetrieb mit dem Betriebspersonal vor Ort vergleichsweise einfach darstellen lässt.

\subsubsection{Operativer Betrieb}

Für den Betrieb eines selbstfahrenden und individuell abrufbaren Personentransportsystems sind trotz des weitestgehend unabhängig operierenden Charakters einige spezielle Bedingungen zu betrachten. So ist beispielsweise Betriebspersonal erforderlich, das, wenn auch nicht notwendigerweise jeden einzelnen Lenk-, Brems- oder Antriebsbefehl, so aber 
doch den grundsätzlichen Betrieb im Rahmen des festgelegten Gebiets (räumliche Begrenzungen) bzw. bestimmter Parameter (z. B. Geschwindigkeit oder Position) sicherstellt. Dafür werden - wie beispielsweise auch bei fahrerlosen Bahnen oder Logistiksystemen Betriebszentren eingerichtet, in denen vergleichsweise wenig Betriebspersonal eine große Anzahl von Fahrzeugen überwacht. Das Betriebszentrum ist mit den einzelnen Fahrzeugen über Funk verbunden und kann die Betriebsdaten des Fahrzeuges überwachen, das Fahrzeug im Notfall stoppen oder auch mit den Passagieren kommunizieren.

In jedem Fall ist aber für den Betrieb der hier betrachteten Fahrzeuge ein Kommunikationssystem einzurichten, das die Betriebsüberwachung ermöglicht. Das kann durch verschiedene Kommunikationsstandards erfolgen, so z.B. über das Mobiltelefonnetz oder aber auch durch WLAN- Netzwerke. In beiden Fällen muss sichergestellt sein, dass die Übertragungszeit und Verfügbarkeit der Datenübertragung den Anforderungen jederzeit gerecht wird.

Damit ist anzumerken, dass aufgrund der kontinuierlichen Funküberwachung und Übernahmemöglichkeit durch das Betriebspersonal keine Vollautomatisierung im strengen Sinne der Definition nach SAE J3016 [1] gegeben ist, da diese für die Vollautomatisierung keinerlei menschliche Überwachung und Übernahme im Ausnahmefall erfordert. Dennoch muss bei Transportsystemen wie dem Navia beachtet werden, dass kein Betriebspersonal unmittelbar vor Ort ist, sodass die Fahrzeuge in der Lage sein müssen, auch einen Notfall selbsttätig zu bewältigen. Das wiederum entspricht einer Vollautomatisierung nach SAE J3016, zeigt aber auch die Grenzen dieser Definition auf.

Des Weiteren sind Streckennetz sowie Betriebszeiten für den Betrieb festzulegen. Das bedeutet, es ist zu definieren, welche Strecken (detaillierte Begrenzung) in welchem Gebiet (übergeordnete Begrenzung) befahren werden können. Die Begrenzung auf ein bestimmtes Gebiet erfolgt aufgrund der Festlegung, welcher Bereich aus Überlegungen hinsichtlich der Nutzerbedürfnisse, Überwachungsanforderungen, Fahrleistungen, Gesetzgebung, Wirtschaftlichkeit oder aus sonstigen Beweggründen heraus mit dem Transportsystem bedient werden soll. Für die hier betrachteten selbstfahrenden und individuell abrufbaren Personentransportsysteme kann die Beschränkung auf eine Fußgängerzone, einen Stadtteil oder auch eine ganze Stadt erfolgen, wobei in allen Fällen im Detail zu beachten ist, auf welchen Wegen, Straßen oder Fahrspuren die Fahrzeuge bewegt werden dürfen.

Für den Betrieb der hier betrachteten Transportsysteme ist für den flexiblen Verwendungszweck zu berücksichtigen, dass auf manchen Wegen, wie beispielsweise in Fußgängerzonen, generell keine motorisierten Fahrzeuge bewegt werden dürfen und in diesem Fall eine entsprechende Genehmigung für den Betrieb dieser speziellen, selbstfahrenden motorisierten Fahrzeuge eingeholt werden müsste. In anderen Fällen, wenn die Fahrzeuge im öffentlichen Straßenverkehr bewegt werden sollen, muss vermutlich in den meisten Gesetzgebungsbereichen eine Erlaubnis für den Betrieb selbstfahrender Fahrzeuge eingeholt werden. In manchen Gesetzgebungsbereichen, wie z.B. in einigen Bundestaaten der USA, ist eine entsprechende Gesetzgebung bereits in der Umsetzung.

Bezüglich der Betriebszeiten ist zu überlegen, ob die Mobilitätsdienstleistung rund um die Uhr angeboten werden soll, was aufgrund des weitestgehend personalunabhängigen 
Betriebs möglich und im Sinne eines kundenfreundlichen Angebots vorteilhaft ist. Allerdings mögen auch hier wieder verschiedene Einschränkungen, vorrangig vermutlich betriebswirtschaftliche Überlegungen, einen Betrieb nur zu bestimmten Zeiten sinnvoll erscheinen lassen. So kann es beispielsweise sein, dass die Fahrzeuge nachts in einem Betriebshof geparkt sein sollten, um Vandalismus vorzubeugen. Eine andere Möglichkeit wäre, dass die Mobilitätsdienstleistung nur dann angeboten werden soll, wenn Busse oder andere öffentliche Verkehrsmittel nicht verkehren, sodass die Fahrzeuge dann tagsüber in einem Betriebshof geparkt wären.

Für alle diese Fälle sind sowohl die räumliche als auch die zeitliche Begrenzung des Fahrzeugbetriebs vergleichsweise einfach durch Einschränkungen mit entsprechenden Betriebsparametern möglich. Das bedeutet, dass mittels der digitalen Karten und auch der freigegebenen Betriebszeiten eine Eingrenzung des Betriebs entsprechend den Vorgaben des Betreibers erfolgen kann.

Um zu gewährleisten, dass das selbstfahrende und individuell abrufbare Personentransportsystem entsprechend den Herstellervorgaben betrieben wird, sind vor der ersten Inbetriebnahme Systemeinrichtung, Personalschulung und -zertifizierung durchzuführen. Das bedeutet, dass das Transportsystem entsprechend den einzuhaltenden Betriebsparametern eingestellt werden muss, was i.A. einen manuellen Betrieb erfordert, bei dem - wie zuvor beschrieben - eine digitale Karte des Betriebsgebiets erstellt wird, sodass die Fahrzeuge während des späteren operativen Betriebs eine akkurate Positionierung vornehmen können.

Zusätzlich ist das Betriebspersonal entsprechend den generellen Fahrzeugeigenschaften sowie bezüglich des speziellen Betriebs zu schulen, d.h., Systemeigenschaften, Betriebsbedingungen, Einschränkungen und andere Charakteristiken müssen vom Betriebspersonal erlernt und verstanden werden. Darüber hinaus ist vor jedem Fahrzeugbetrieb, beispielsweise zu Beginn eines jeden neuen Betriebstages, die Betriebssicherheit ähnlich wie bei Flugzeugen festzustellen, aber auch Sonderfälle im Betrieb wie beispielsweise abweichende Streckenführungen müssen erkundet und im Betriebsprotokoll des Transportsystems festgehalten werden.

Aus diesen Gegebenheiten ist zu erkennen, dass, obwohl die Fahrzeuge selbstfahrend sind, eine beträchtliche Vorbereitung für den Betrieb erforderlich ist, die nicht automatisiert erfolgen kann, sondern durch das Betriebspersonal erfolgen muss. Dennoch ist anzunehmen, dass für den Gesamtbetrieb des Transportsystems deutlich weniger Personal erforderlich ist, als wenn für jedes Fahrzeug zu jedem Zeitpunkt ein individueller Fahrer eingesetzt werden müsste.

\subsubsection{Geschäftsmodell}

Einer der Hauptgründe für den Einsatz von selbstfahrenden und individuell abrufbaren Personentransportsystemen ist der deutlich geringere Personalaufwand im Vergleich zum Betrieb mit konventionellen Fahrzeugen. Dies hat auch Einfluss auf das Geschäftsmodell. Der Anbieter des Navia-Systems gibt an, dass die Betriebskosten für den Personentransport 
damit um ca. 50 Prozent gesenkt werden können [7]. Das Geschäftsmodell für derartige Systeme besteht also darin, die Mobilitätsleistung durch die Einsparung von Personal deutlich günstiger anzubieten als beispielsweise mit einem Taxidienst. Gegenüber dem individuell genutzten Pkw ergibt sich der Vorteil, dass der Nutzer nicht auch die Fahraufgabe übernehmen muss, sondern anderen Beschäftigungen wie Arbeit, Freizeit oder Ausruhen nachgehen kann.

Darüber hinaus können keine weiteren betriebswirtschaftlichen Vorteile ausgewiesen werden, da ein individuell abrufbarer Betrieb auch mit konventionellen, von Menschen gesteuerten Fahrzeugen möglich wäre. Der Vorteil der selbstfahrenden Fahrzeuge ist allerdings, dass sie auch zu betriebswirtschaftlich unattraktiven Zeiten betrieben werden können, so z. B. nachts, wenn vielleicht nur einmal pro Stunde eine Fahrt von zehn Minuten Dauer angefordert wird. Ein Fahrer müsste dabei 50 Minuten warten, ohne weitere Einnahmen zu haben. Wenn dagegen das selbstfahrende Fahrzeug für 50 Minuten steht, wäre die Wartezeit unerheblich, solange die Gesamtnutzung für den betriebswirtschaftlichen Betrachtungszeitraum profitabel ist.

Bisher sind die Transportsysteme allerdings nur im Pilotbetrieb eingesetzt worden, so beispielsweise zu eingeschränkten Zeiten in Universitäts- oder Einkaufszentren [3, 4, 5]. Da diese Einsätze vorrangig der Vorführung des Systemkonzepts galten, kann das Geschäftsmodell noch nicht vollständig bewertet werden. Mit einem projektierten Einsatz der Fahrzeuge im kommerziellen Betrieb ab 2015 werden dann das Geschäftsmodell sowie Umsatzziele der Betreiber und Hersteller dieser Transportsysteme bewertbar sein.

\subsection{Erfahrungen bei der Umsetzung des Transportsystems}

Der folgende Abschnitt soll einen Überblick über die Erfahrungen bei der Umsetzung des selbstfahrenden und individuell abrufbaren Personentransportsystems „Navia“ der Firma Induct an der Stanford Universität geben. Da die Fahrzeuge bis Mitte 2014 nur sporadisch im Einsatz waren, ist dies ein erster Erfahrungsbericht, der an anderer Stelle und zu späterer Zeit fortgesetzt werden soll. Allerdings können jetzt schon Erfahrungen über die Vorgehensweise bei der Einführung vermittelt werden, die für andere Wissenschaftler und Verkehrsplaner bei der Umsetzung ähnlicher Transportsysteme hilfreich sein können.

\subsubsection{Evaluations-, Versuchs- und Öffentlichkeitsbetrieb}

Für die hier dargestellten Fahrzeuge werden entsprechend Tab. 14.1 verschiedene Betriebsphasen betrachtet, um das Transportsystem zunächst in einer Evaluationsphase zu klassifizieren, dann in einer Versuchsphase zu optimieren und schließlich im Öffentlichkeitsbetrieb anzubieten.

Zunächst müssen generelle Erfahrungen mit den Fahrzeugen gesammelt werden, da ein derartiges Transportsystem noch weitestgehend unbekannt und die Risikobewertung damit 
Tab. 14.1 Abgrenzung und Charakteristiken der drei Betriebsphasen

\begin{tabular}{|c|c|c|c|}
\hline & Evaluation & Versuch & Öffentlichkeit \\
\hline Zielsetzung & $\begin{array}{l}\text { Risikobewertung, } \\
\text { Eignung f. Forschung }\end{array}$ & $\begin{array}{l}\text { Forschungsprojekt, Betriebs- } \\
\text { optimierung }\end{array}$ & $\begin{array}{l}\text { Personentransport } \\
\text { als Dienstleistung }\end{array}$ \\
\hline Betriebsgebiet & $\begin{array}{l}\text { abgeschlossen, } \\
\text { begrenzt }\end{array}$ & $\begin{array}{l}\text { teilöffentlich, } \\
\text { begrenzt }\end{array}$ & $\begin{array}{l}\text { öffentlich, } \\
\text { begrenzt }\end{array}$ \\
\hline Betriebszeiten & $\begin{array}{l}\text { begrenzt, } \\
\text { Kurzzeitversuch }\end{array}$ & $\begin{array}{l}\text { begrenzt, } \\
\text { Langzeitversuch }\end{array}$ & $\begin{array}{l}\text { unbegrenzt, } \\
\text { prinzipiell } 24 \mathrm{~h}\end{array}$ \\
\hline Nutzergruppe & Projektbeteiligte & $\begin{array}{l}\text { ausgewählte } \\
\text { Versuchsteilnehmer }\end{array}$ & $\begin{array}{l}\text { generelle } \\
\text { Öffentlichkeit }\end{array}$ \\
\hline Überwachung & $\begin{array}{l}\text { Betriebspersonal } \\
\text { am Fahrzeug }\end{array}$ & $\begin{array}{l}\text { Betriebspersonal am Fahrzeug, } \\
\text { Funkverbindung }\end{array}$ & $\begin{array}{l}\text { Betriebspersonal in } \\
\text { Funkverbindung }\end{array}$ \\
\hline
\end{tabular}

schwierig ist. Gleichzeitig ergeben sich weitere Fragestellungen für die Forschung. Das hier verwendete System ermöglicht einen sehr flexiblen Betrieb für die entsprechenden Belange. Für den anfänglichen Evaluationsbetrieb ist es vorteilhaft, dass die Fahrzeuge relativ einfach auf ein gewünschtes Betriebsgebiet eingerichtet und dann vom Betriebspersonal überwacht werden können. Dabei ist es sehr hilfreich, dass die Fahrzeuge kaum eine spezielle Infrastruktur benötigen, sondern nach dem Einrichten weitgehend autark operieren.

Der nachfolgende Versuchsbetrieb, in dem entsprechend den Zielsetzungen des Autonomous Systems Laboratory [8] die räumliche sowie zeitliche Verteilung selbstfahrender und individuell abrufbarer Fahrzeuge zu optimieren ist, soll dann möglichst realitätsnah erfolgen. Das bedeutet, dass Nutzer des Systems sich trotz des Versuchsbetriebs wie im Fall eines realen Mobilitätsbedarfs verhalten können. Also sollen sie beispielsweise einen Fahrtgrund haben, warum sie mit einer möglichst kurzen Warte- und Fahrtzeit von einem Ort zum anderen kommen wollen. Auch dieser Betrieb ist mit dem verwendeten Transportsystem recht flexibel und zielgerichtet darzustellen, da die angebotenen Fahrtrouten sowie Haltestellen vergleichsweise einfach zu programmieren sind und damit die Anforderungen für den Versuchsbetrieb effizient umgesetzt werden können.

Der Versuchsbetrieb würde schließlich in den eigentlichen Öffentlichkeitsbetrieb übergehen, in dem bis auf Weiteres keine wissenschaftlichen Untersuchungen oder Optimierungen mehr geplant wären, sondern der Betrieb entsprechend den Ergebnissen aus dem Versuchsbetrieb umgesetzt wird. Das hier verwendete Transportsystem ermöglicht diese schrittweise Einführung, da das Betriebsgebiet und die Betriebszeiten recht einfach festzulegen sind und aufgrund der geringen Fahrgeschwindigkeit und Fahrstrecke eine Betriebsüberwachung problemlos ist. 


\subsubsection{Auswahl des Fahrzeugkonzepts}

Um den Anforderungen für den Versuchsbetrieb gerecht zu werden, ist ein vollautomatisiertes, d.h. ohne Fahrer operierendes Fahrzeug erforderlich, das innerhalb gegebener Grenzen selbstfahrend ist und von Nutzern individuell abgerufen werden kann. Zusätzlich ist eine offene Betriebsarchitektur erforderlich, sodass die Bereitstellung und Verteilung der Fahrzeuge für den Versuchsbetrieb variiert werden können. Die Fahrzeuge selbst müssen nicht notwendigerweise über eine offene Architektur verfügen; das Betriebssystem der individuellen Fahrzeugsteuerung oder die Umfelderkennung sind nicht Gegenstand der Forschungsarbeiten und brauchen deswegen auch nicht weiter beeinflusst oder gar verändert zu werden.

Die von der Firma Induct angebotenen Fahrzeuge „Navia“ mit der zugehörigen Betriebsarchitektur erfüllen diese Anforderungen. Zusätzlich liegen bereits Erfahrungen von anderen Implementierungen dieses Mobilitätskonzepts vor [3, 7], was einen stabilen Versuchsbetrieb erwarten lässt. Darüber hinaus hat Induct seine Schutzrechte bezüglich des Navia vorwiegend auf dem Gebiet der Umfelderkennung und Erstellung des Referenzsystems für die Fahrzeugautomatisierung, sodass die hier betrachtete Forschungsarbeit auf dem Gebiet des übergeordneten Fahrzeugbetriebs zur optimalen Nachfragebedienung kaum eine Überschneidung darstellt, sondern vielmehr eine Ergänzung. Aus diesen Gründen heraus wurde das Induct Navia-Konzept für die Arbeiten des Autonomous Systems Laboratory an der Stanford Universität [8] für den Versuchsbetrieb in Betracht gezogen und entsprechend den in den nachfolgenden Abschnitten benannten Kriterien bewertet.

\subsubsection{Risikobewertung und Rechtseinordnung}

Die Einordnung in das Rechtssystem erfolgt für den hier betrachteten Einsatzfall schrittweise. Da die Fahrzeuge zunächst für die Evaluation in nicht allgemein zugänglichen Gebieten betrieben werden, gelten dort Sonderregelungen bezüglich Fahrzeugbetrieb und Betriebshaftung. Diese konnten an der Stanford Universität im Einvernehmen mit der Abteilung für Risikobewertung sowie der Rechtsabteilung formuliert werden. Die Haftung wird im Wesentlichen beim Betreiber der Fahrzeuge belassen, was je nach Betriebszustand das im bzw. am Fahrzeug anwesende oder über Funküberwachung verbundene Betriebspersonal ist. Der Betrieb der Fahrzeuge wird mit entsprechenden Auflagen der internen (d.h. der Stanford Universität angehörigen) Stellen für Risikobewertung zugelassen.

Die Risikobewertung für den hier betrachteten Betriebsfall ist deutlich einfacher planbar als dies für den öffentlichen Straßenverkehr der Fall ist, da sowohl die Fahrgeschwindigkeit als auch das Einsatzgebiet deutlich begrenzt sind. Das bedeutet, dass anders als bei konventionellen Pkw, die deutlich schneller und prinzipiell überall fahren können, die Begrenzung auf maximal $20 \mathrm{~km} / \mathrm{h}$ und ein eng umrissenes Gebiet zu einem vergleichsweise geringen Betriebsrisiko führen. Im Einsatzgebiet wird für den hier betrachteten Fall zunächst nur der Betrieb unter Ausschluss der Öffentlichkeit bzw. unter direkter Aufsicht durch das Betriebspersonal, unmittelbar im oder am Fahrzeug, zugelassen. Diese Beschrän- 
kungen erlauben es, über erste Schritte beim Betrieb der Fahrzeuge allen Projektbeteiligten ein Verständnis für die Möglichkeiten, Beschränkungen und weiteren Eigenschaften zu vermitteln. Damit können dann schrittweise die Risikobewertung und Rechtseinordnung vorgenommen und letztlich auch der eigentliche Versuchsbetrieb ausgeweitet werden. Das bedeutet, dass Risikobewertung und Rechtseinordnung aufgrund des neuen und weitgehend unbekannten Charakters der Fahrzeuge am praktischen Beispiel erfolgen und kaum im Voraus festgelegt werden können.

Mit der zunehmenden Ausweitung des Betriebes auf den Versuchs- und dann den Öffentlichkeitsbetrieb (s. Tab. 14.1) gelten zunehmend öffentliche Gesetzgebungen, sofern die Fahrzeuge auf öffentlichen Straßen betrieben werden. Für den hier betrachteten Anwendungsfall gelten die kalifornischen Regelungen, die in der ersten Hälfte des Jahres 2014 noch in einer abschließenden Gesetzgebungsphase waren. Dabei ist besonders anzumerken, dass ab September 2014 nur noch Fahrzeughersteller Versuche mit ,autonomen“ (also selbstfahrenden oder vollautomatisierten) Fahrzeugen vornehmen dürfen [9]. Da als „Fahrzeughersteller“ definiert wird, wer ein Fahrzeug zu einem „,autonomen“ Fahrzeug verändert, wird an dieser Stelle zu sehen sein, auf welchen Verkehrswegen die Fahrzeuge an der Stanford Universität bewegt werden und wer der Fahrzeughersteller im Sinne der kalifornischen Gesetzgebung ist. Solange die Fahrzeuge von der Stanford Universität nicht im Sinne der Automatisierung verändert, sondern lediglich Daten über deren Position, Zeitverhalten und Nutzeranforderungen ausgewertet und optimiert werden, wird erwartet, dass nicht die universitären Wissenschaftler als der Fahrzeughersteller im Sinne des Gesetzes anzusehen sind, sondern der eigentliche Hersteller der Fahrzeuge, von dem die Stanford Universität diese bezogen hat.

Allerdings ist die Gesetzeslage derzeit schwer vorherzusagen, da sie sich noch in der Entstehungsphase befindet. Es ist zu erwarten, dass auf den verschiedensten gesetzgebenden Ebenen (d.h. auf bundesstaatlichen, kommunalen, aber auch nationalen Ebenen) noch Veränderungen auftreten werden, auf die zu reagieren sein wird. Generell scheint sich für das hier betrachtete Betriebsgebiet, d. h. eine innovative Universität in Kalifornien, eine sehr positive Grundhaltung der Verantwortlichen und der Öffentlichkeit gegenüber automatisierten Fahrzeugen zu ergeben. Und auch die gesetzgebenden Organe scheinen eher eine unterstützende und wohlwollende Haltung einzunehmen. Es ist auch zu verspüren, dass im Einzelfall Sonderregelungen erreicht werden können, um einen Betrieb zu ermöglichen, der durch die Gesetzeslage möglicherweise nicht eindeutig definiert ist. Eine derartige Grundhaltung wird auch in anderen Teilen der USA beobachtet, sodass die Gesetzgebung kein grundsätzliches Hindernis bei der Einführung automatisierter Fahrzeuge zu sein scheint, sondern eher eine zusätzliche Überprüfungsinstanz.

\subsubsection{Vertragsgestaltung}

Für den Betrieb des selbstfahrenden und individuell abrufbaren Transportsystems ist ein Vertrag erforderlich, der im Wesentlichen die Rechtsbeziehung zwischen dem Eigentümer 
des Fahrzeugs (für den hier betrachteten Versuchsbetrieb ist das der Hersteller) und dem Nutzer (hier die Stanford Universität als Forschungsbetreiber) festlegt. Darin eingeschlossen sind Rechte und Pflichten bezüglich der generellen Nutzung, Information und Kommunikation, Wartung, Haftung und anderer Bestimmungen für den Versuchsbetrieb.

Im vorliegenden Fall wird ein Leihvertrag für ein selbstfahrendes Fahrzeug zwischen dem Eigentümer und dem Nutzer des Fahrzeugs geschlossen, der zunächst nur für die Evaluationsphase zur Vorbereitung eines eventuellen Forschungsprojekts gilt. Sobald ein konkretes Forschungsprojekt mit dem selbstfahrenden und individuell abrufbaren Personentransportsystem verfolgt würde, wäre ein neuer oder eventuell auch zusätzlicher Vertrag notwendig, der dann für den konkreten Anwendungsfall alle zuvor genannten Gegebenheiten regelt sowie zusätzlich beispielsweise die Möglichkeiten, für Forschungszwecke in die Umfelderkennung und Routenplanung einzugreifen, was für die Evaluationsphase nicht vorgesehen ist.

In dem für die Evaluationsphase eingesetzten Vertrag zwischen Eigentümer und Nutzer der Fahrzeuge wird die Haftung für den Betrieb dem Betreiber auferlegt. Dabei ist der Betreiber die Rechtsperson, die den Betrieb eines solchen Fahrzeugs initiiert und überwacht, also entweder das Betriebspersonal im oder am Fahrzeug oder das Betriebspersonal, welches über eine Funkverbindung mit dem Fahrzeug verbunden ist.

\subsubsection{Auswahl des Betriebsgebiets und der Betriebsszenarien}

Das Betriebsgebiet für das selbstfahrende und individuell abrufbare Personentransportsystem ist zunächst räumlich zu begrenzen. Für den anfänglichen Evaluations- und anschließenden Versuchsbetrieb mit dem Transportsystem ist weniger die Größe des Betriebsgebiets von Interesse, als vielmehr die Vielfalt der Nutzer, Anwendungsfälle und Betriebssituationen. Diese können für den Evaluations- und Versuchsbetrieb auch proaktiv als Bestandteil eines Versuchsablaufs dargestellt werden, d. h., Beteiligte des Versuchsprojekts können verschiedene Situationen darstellen, sodass das Verhalten der Fahrzeuge beobachtet und bewertet werden kann. Damit kann ein kleines Betriebsgebiet durchaus ausreichen, um eine Vielzahl von Situationen und Betriebszuständen bewerten zu können.

Da es sich bei dem selbstfahrenden und individuell abrufbaren Personentransportsystem um ein neuartiges Konzept handelt, dessen Risikobewertung nur begrenzt auf der Grundlage von Erfahrungswerten erfolgen kann, muss das Einsatzgebiet so ausgesucht werden, dass zunächst ein realitätsnaher, aber doch vollständig kontrollierbarer Einsatz sichergestellt ist. Das bedeutet, dass entsprechende Nutzer die Fahrzeuge innerhalb des Betriebsgebietes individuell abrufen, ein Fahrziel eingeben und das Fahrzeug die entsprechende Fahraufgabe ohne direkten Einfluss des Betriebspersonals ausführt. Dabei muss das Betriebspersonal aber die Fahrzeuge überwachen können, und es sollte für diesen anfänglichen Versuchsbetrieb auch Kontrolle oder zumindest Wissen darüber haben, welche Personen und Fahrzeuge sich in dem Betriebsgebiet aufhalten und somit mit den Fahrzeugen interferieren könnten. 
Die Übersicht über die Personen im Betriebsgebiet ist wichtig, um das Potenzial für Kollisionen oder sonstige Störungen zu erkennen und damit zu minimieren. Beispielsweise sollten sich zu Beginn keinerlei Personen im Betriebsgebiet der Fahrzeuge aufhalten, die nicht mit dem Transportsystem vertraut sind bzw. sich nicht entsprechend den geltenden Vorgaben verhalten (z. B. vor das fahrende Fahrzeug treten). Vielmehr muss sichergestellt sein, dass alle Personen mit den Charakteristiken und Risiken des Transportsystems vertraut sind, sich aber dennoch wie reale Nutzer verhalten, die die Fahrzeuge anfordern und Fahrziele eingeben. Dabei kann in Betracht gezogen werden, dass alle Personen, die mit den Fahrzeugen interagieren, ihre Einverständniserklärung abgeben, dass sie mit den Eigenschaften und Beschränkungen vertraut sind und ihr eigenes Verhalten dementsprechend ausrichten werden.

Nach einem anfänglich stark eingeschränkten Betriebsgebiet, welches aufgrund der zuvor beschriebenen Gründe eventuell sogar eingezäunt und nur mit besonderer Genehmigung zugänglich sein mag, sollte der Betrieb auf ein zwar überwachtes, aber ansonsten öffentliches Betriebsgebiet erweitert werden, damit eine große Anzahl von realen Nutzern ermöglicht wird. Das bedeutet, dass die Nutzer in dieser Phase keine Vorkenntnis bezüglich des Systems haben und auch keine gesonderte Einverständniserklärung abgeben, sondern die Fahrzeuge genauso benutzen wie beispielsweise ein Taxi oder einen Bus. Dafür ist es wichtig, dass das Betriebsgebiet einen Bereich beschreibt, in dem die Nutzer einen Mobilitätsbedarf haben, für den die hier betrachteten Fahrzeuge aufgrund der Reichweite, Fahrgeschwindigkeit und Transportkapazität eine reale Lösung darstellen.

Eine Universität wie Stanford hat die Möglichkeit, die hier beschriebenen Anforderungen umzusetzen: Der Universitätscampus hat eine Ausdehnung, die für ein Fahrzeug wie den Navia befahrbar ist; der Transportbedarf für Personen und Gegenstände ist hoch; die Verkehrsbelange werden weitestgehend von der zuständigen Abteilung der Universität (Parking \& Transportation Services) koordiniert. Damit kann für den anfänglichen Evaluationsbetrieb ein abgegrenztes Gebiet ausgewiesen werden, zu dem nur Versuchsbeteiligte Zugang haben. In der nächsten Phase können dann beispielsweise Fußgängerzonen und Straßen ausgewiesen werden, auf denen die Fahrzeuge betrieben werden. In allen Fällen lässt sich sicherstellen, dass die erforderlichen Kommunikationssysteme zur Überwachung durch das Betriebspersonal zur Verfügung stehen und das Personal bei Bedarf Zugang zu den Fahrzeugen hat.

Nachdem bekannt geworden war, dass die Navia-Fahrzeuge an der Stanford Universität eingesetzt werden sollen, ergeben sich sehr schnell mehrere Anfragen von interessierten Abteilungen, das Transportsystem einzusetzen. Diese Anfragen kommen von den Betreibern der lokalen Busse, um eine punktuelle und flexible Erweiterung des Linienbetriebs anzubieten; von den Versorgungs- sowie Instandsetzungsabteilungen, um einen flexiblen und personalungebundenen Transport von Gegenständen auf dem Campus zu ermöglichen; sowie von den Betreibern einer separaten Forschungseinrichtung, um einen Personentransport auf dem abgegrenzten Forschungscampus einzurichten.

Unter Berücksichtigung der vorhandenen Anfragen und Möglichkeiten wird zunächst der Betrieb auf einem zugangsgeregelten Parkplatz verfolgt, wo verschiedene Betriebs- 
szenarien nachgestellt werden können. Danach wird der realitätsnahe Einsatz auf dem Gelände der separaten Forschungseinrichtung geplant, wo eine größere Zahl realer Nutzer erwartet wird und jederzeit eine vollständige Überwachung des Betriebs gewährleistet werden kann.

Zu Beginn des Evaluationsbetriebs sind dann spezielle Szenarien abzubilden, die die Risikobewertung und entsprechende Sicherheitstests ermöglichen. Dafür sind die Fahrzeuge beispielsweise mit Hindernissen und anderen Objekten zu konfrontieren. Es sollen reale sicherheitsrelevante Situationen abgebildet werden, in denen die Fahrzeuge sich in geforderter Art und Weise so verhalten, dass sich keine kritischen Betriebszustände ergeben. Die Objekte, mit denen die Fahrzeuge konfrontiert werden, sind Fußgänger, Radfahrer, Fahrzeuge aller Art, Tiere und ganz allgemein Gegenstände, die es in dem Betriebsgebiet gibt, wie beispielsweise Mülltonnen, Pakete, Kartons, Arbeitsmaterial, Pflanzen, Gebäude usw.

Dabei sind stationäre von beweglichen Objekten zu unterscheiden, da erstere in der digitalen Umgebungskarte definiert werden können und die Bahn der Fahrzeuge dementsprechend vorgegeben werden kann. Bewegliche Objekte - in diesem Fall auch solche Objekte, die nur selten und nur durch Fremdeinwirkung bewegt werden können wie z. B. ein Schuttcontainer - müssen vollständig von der Umfeldsensorik erfasst werden, um eine Kollision zu vermeiden. Je nach Eigenart des Objekts sind Betriebsszenarien zu definieren, sodass ein für die Risikobewertung und für Sicherheitstests relevantes Verhalten dargestellt wird: Personen und Fahrzeuge sollten relativ kurzfristig und schnell vor das Fahrzeug gelangen können, Mülltonnen und Pakete eher langsamer. Für alle diese Objekte ist das Verhalten der Fahrzeuge dahingehend zu bestimmen, inwieweit das Objekt rechtzeitig und zuverlässig genug erkannt und eine Kollision vermieden wird.

\subsubsection{Einrichtung des Transportsystems und Zertifizierung des Personals}

Der Hersteller der Fahrzeuge legt fest, wie das Transportsystem mit den selbstfahrenden Fahrzeugen einzurichten ist. Dazu erfolgt zunächst die Festlegung der Route, die dann mit einem der Fahrzeuge in einem speziellen Betriebsmodus befahren wird, um die digitale Karte des Betriebsgebiets zu erstellen. Die Betriebsdaten werden anschließend editiert, um stationäre und bewegliche Hindernisse zu kategorisieren. Der Hersteller stellt abschließend über die Einsatzart und das Betriebsgebiet ein Dokument aus, das die Parameter und Begrenzungen definiert und damit den zulässigen Betrieb auch für eventuelle Haftungsfragen genau festgelegt. Diese Festlegungen werden in einem Zertifikat des Herstellers dokumentiert.

Zur Zertifizierung des Betriebspersonals erfolgt eine Einweisung durch den Hersteller, bei der operative Anforderungen, funktionale Merkmale, Betriebsarten, technische Details, Einschränkungen, Risiken usw. erklärt werden und das Verständnis des Systems im praktischen Umgang erlernt und dann auch abgeprüft werden. Der Hersteller dokumentiert die Zertifizierung und beurkundet damit, welche Personen die Ausbildung absolviert haben 
und mit dem System vertraut sind. Festgelegt ist auch anhand der drei Stufen „Betrieb“, „Einrichtung“ und „Wartung/Modifikation“, ob die Personen das System lediglich betreiben, für den Betrieb einrichten oder auch warten und verändern dürfen.

Diese Abläufe und Umfänge müssen zunächst vom Hersteller der Fahrzeuge vorgegeben sein, da dieser die beste Kenntnis über das System sowie deren Risiken hat. Um eventuelle Besonderheiten der Betriebsumgebung zu berücksichtigen, sollte allerdings der Betreiber des Systems (d.h. nicht der Hersteller, sondern der verantwortliche Beobachter vor Ort oder in Funkverbindung) Änderungen oder Erweiterungen für Systemtest, Wartung und Zertifizierung vornehmen, da hier Sondersituationen am ehesten offenbar werden. Wenn in Zukunft derartige selbstfahrende und individuell abrufbare Transportsysteme weitere Verbreitung erfahren und damit die Gesetzgeber auch mehr Erfahrung gesammelt haben, sind entsprechende Abläufe und Umfänge für die Zertifizierung von Fahrzeug und Personal wahrscheinlich eher vom Gesetzgeber oder von beauftragten Institutionen zu erstellen.

\subsubsection{Systemstart und Betriebsüberwachung}

Für den eigentlichen Betrieb der Fahrzeuge gibt der Hersteller einen Systemstart mit einer entsprechenden Systemüberprüfung vor. Dabei wird eine Vielzahl von Fahrzeug- und Umgebungsparametern überprüft, bevor die Freigabe für den eigentlichen Betrieb erfolgt. Der vorgegebene Ablauf stellt einen guten Kompromiss aus Detailgenauigkeit und Handhabbarkeit dar, sodass vor jedem Betrieb Gewissheit besteht, dass sowohl das Transportsystem als auch die Betriebsumgebung den Sicherheits- und Funktionsanforderungen entsprechend dem zertifizierten Betrieb genügen. Ebenso sind Wartungsumfänge mit verschiedenen Intervallen vorgegeben, die Sicherheit und Funktion langfristig sicherstellen sollen.

Für den Betrieb der Fahrzeuge im Falle einer Funküberwachung (d.h. kein Betriebspersonal befindet sich unmittelbar im oder am Fahrzeug) sieht der Hersteller eine redundante Kommunikation vor. Dazu werden zwei voneinander unabhängige drahtlose Kommunikationssysteme, beispielsweise Mobiltelefon- und WLAN-Netz oder zwei unabhängige Mobiltelefonnetze, implementiert, über die das Betriebspersonal Fahrzeugdaten abrufen, gegebenenfalls einen Nothalt einleiten oder auch mit den Insassen des Fahrzeugs kommunizieren kann. Die genauen Überwachungsumfänge, beispielsweise Position, Geschwindigkeit, Fahrtrichtung, Insassen und Türverriegelung oder aber auch die komplette Videoüberwachung der Umgebung, sind individuell festzulegen, jedoch derzeit (Juni 2014) noch nicht genau bestimmt.

Bisher wurden die Fahrzeuge an der Stanford Universität nur mit dem Betriebspersonal unmittelbar im oder am Fahrzeug und mit direktem Zugriff auf einen Notfallschalter betrieben. Aus diesem Grund liegen noch keine erweiterten Erfahrungen zum Betrieb mit Funküberwachung vor. Allerdings ist bereits aus dieser begrenzten Erfahrung die Wahrscheinlichkeit zu erkennen, dass eine Kollision eher auf einen anderen Verkehrsteilnehmer (Fußgänger, Radfahrer, anderes Fahrzeug) zurückzuführen ist als auf das Fahrzeug selbst. 
Die Kombination der verwendeten Umfelderkennung, Objektklassifizierung und Routenbestimmung ist also geeignet - ganz besonders in Anbetracht der geringen Fahrgeschwindigkeit und des bekannten sowie begrenzten Betriebsumfelds -, um das Fahrzeug sehr sicher und störungsfrei zu führen.

Für den späteren Betrieb mit Funküberwachung wird zu bewerten sein, wie schnell das Betriebspersonal im Störungsfall vor Ort sein könnte. So könnte es vorkommen, dass die vorprogrammierte Route durch Baumaßnahmen oder auch widerrechtlich geparkte Fahrzeuge versperrt ist. In solch einem Fall würden die Fahrzeuge den Betrieb selbsttätig unterbrechen und die Betriebsstelle informieren. Je nachdem, wo das Betriebspersonal dann lokal angesiedelt ist, wird eine Zeit in der Größenordnung von Minuten oder auch deutlich länger vergehen, bis jemand vor Ort sein wird, um die Störung zu beheben. Dieses Modell ist dem Betrieb von Fahrstühlen entnommen, wo die Notruftaste auch einen sofortigen Kontakt zu einer Betriebszentrale herstellt, das Betriebspersonal möglicherweise aber eine längere Zeit benötigt, bis es vor Ort sein kann. Wie die Nutzer und Insassen des selbstfahrenden und individuell abrufbaren Personentransportsystems damit umgehen bzw. ob diese Zeitdauer ein Problem darstellt, ist im späteren Öffentlichkeitsbetrieb zu ermitteln. Im Unterschied zum Fahrstuhl ist aber festzuhalten, dass die Passagiere des Transportsystems aufgrund der offenen Bauweise zu jeder Zeit direkten Kontakt zur Umgebung haben und die Fahrzeuge (s. Abb. 14.2) im Notfall auch vergleichsweise einfach verlassen können.

\subsubsection{Information für Nutzer und Passanten}

Da es sich bei dem selbstfahrenden und individuell abrufbaren Personentransportsystem um eine Neuerung im Personentransport handelt, müssen die Nutzer über die Möglichkeiten und Risiken aufgeklärt werden. Zunächst sollte der normale Betrieb erläutert werden, d.h. die eigentliche Bedienung ist zu beschreiben. Hierfür sollte nur ein Mindestmaß an Beschreibung vorgesehen werden, da das Transportsystem eine ähnliche Nutzung wie ein Taxi oder Bus ermöglichen soll, die beide auch keine Beschreibung erfordern. So ist eher auf die Risiken und auf die speziellen Aspekte des Versuchsbetriebs hinzuweisen. Das beinhaltet eine Aufklärung über die Grenzen des Systems und dessen Gefahren, wie beispielsweise die Möglichkeit, dass ein Objekt nicht rechtzeitig erkannt wird, aber auch das Verhalten im Ausnahmefall.

Die Nutzer für den Evaluations- und Versuchsbetrieb werden über die Eigenschaften und Risiken in einer schriftlichen Information aufgeklärt. Dazu gehört auch die Information, an wen sich die Nutzer in einem eventuellen Schadensfall als rechtlich Verantwortlichen wenden sollen. Ähnlich sind im späteren Öffentlichkeitsbetrieb Möglichkeiten vorzusehen, dass die Nutzer dem Betreiber Rückmeldung und Fragen zukommen lassen können. Da es sich um ein neues Transportsystem handelt, ist die Nutzerbewertung von großer Bedeutung; deswegen ist es wichtig, dass die Nutzer wissen, an wen sie sich wenden können.

Ebenso wie die eigentlichen Nutzer des selbstfahrenden Transportsystems müssen auch Passanten und andere Verkehrsteilnehmer über den Betrieb der Fahrzeuge aufgeklärt 
werden. Zwar ist nicht unbedingt Wissen über die Nutzung erforderlich, angesichts der Interaktion zwischen den Fahrzeugen und anderen Verkehrsteilnehmern müssen diese aber über die Möglichkeiten und Risiken in Kenntnis gesetzt werden. Das schließt ganz besonders auch Vorfahrtsregelungen oder Sonderregelungen mit ein. Passanten und andere Verkehrsteilnehmer sollten wissen, wie sie die Fahrzeuge im Notfall gegebenenfalls von außen stoppen können, indem sie den Notfallschalter betätigen. Auch sollte den Passanten der offizielle Betreiber der Fahrzeuge bekannt sein, an den sie sich mit Rückmeldungen und Fragen wenden können.

\subsection{9 Öffentliche Reaktionen}

Auch wenn das selbstfahrende und individuell abrufbare Personentransportsystem bisher nur in begrenztem Maße in der Öffentlichkeit an der Stanford Universität zu sehen war (das war bislang nur der Fall, wenn das Fahrzeug vom Betriebspersonal gesteuert zu einem anderen Betriebsgebiet überführt wurde), kann über die öffentlichen Reaktionen doch schon einiges ausgesagt werden. Diese Beobachtungen wurden bei Überführungsfahrten und Besuchen beim Evaluationsbetrieb, aber auch an der Parkposition der Fahrzeuge gemacht.

Dabei sei ganz besonders betont, dass es sich bei den folgenden Beschreibungen nicht um eine methodische Studie handelt, sondern eher um zufällige Beobachtungen, die für weitere Untersuchungen Anregung geben sollen.

Generell gilt, dass die Öffentlichkeit, also alle Unbeteiligten an diesem Projekt, sehr positiv auf das Fahrzeug reagieren. Das mag zwei Hauptursachen haben:

Zum einen scheinen unbeteiligte Passanten oder Betrachter generell sehr interessiert und neugierig in Bezug auf automatisierte Fahrzeuge zu sein. Das liegt möglicherweise daran, dass derartige Technologien derzeit sehr intensiv in der allgemeinen Medienberichterstattung behandelt werden, aber auch in populärwissenschaftlichen Beiträgen und Fachartikeln ein zentrales Thema sind. Vor allem die allgemeinen Medien berichten häufig sehr positiv und zuweilen sogar euphorisch über ,autonome“ Fahrzeuge, bei denen der Mensch die Fahraufgabe komplett an den Computer übergibt und zwischen Start und Ziel anderen Tätigkeiten nachgehen kann. Wenn Menschen unter diesem Eindruck zum ersten Mal ein solches Fahrzeug sehen, sind Neugierde, Offenheit und Vertrauensvorschuss vermutlich naheliegende Reaktionen, die immer wieder beobachtet werden konnten.

Zum anderen trägt der Charakter des hier betrachteten Fahrzeugs zu einer spontanen, positiven Reaktion von Passanten und Betrachtern bei. Das Fahrzeug hat eine sehr offene Bauweise (s.Abb. 14.2), so z. B. offene Seitenwände ohne Fenster oder eine offene Reling anstelle einer geschlossenen Tür. Durch diese Bauweise sind die Insassen des Fahrzeugs klar zu erkennen und für Passanten wahrnehmbar, was einen positiven Eindruck vermittelt. Das bedeutet, das Erscheinungsbild ist ganz anders, als wenn das Fahrzeug beispielsweise getönte Scheiben und geschlossene Türen hätte, sodass Passanten nicht wüssten, wer in dem selbstfahrenden Fahrzeug unterwegs ist. Ähnliches kann beispielsweise im öffentli- 
chen Straßenverkehr beobachtet werden, wenn man die Eindrücke vergleicht, die beispielweise eine verdunkelte Limousine oder ein offenes Cabrio vermitteln. Für das hier betrachtete Fahrzeug kommt hinzu, dass das Design Elemente aus dem Bootsbau aufgreift, so beispielsweise weißen Kunststoff für den Aufbau, beigefarbenes Kunstleder für die Passagierplätze, helles Teakholz für den Fußboden sowie ein nahezu segelartiges Dach. Die Reaktionen von Betrachtern des Fahrzeugs reichen von „Landyacht“ bis hin zu „Whirlpool auf Rädern" - alles positive Assoziationen, die den ersten Eindruck bestimmen.

Darüber hinaus ist das Fahrzeug vergleichsweise langsam; es operiert oft kaum schneller als mit Schrittgeschwindigkeit. Dadurch bekommen Passanten den Eindruck von Sicherheit: Fußgänger empfinden, dass sie zur Not schnell beiseitetreten könnten; Autofahrer fühlen sich diesem Fahrzeug wahrscheinlich ,überlegen“, was auch an dessen weitestgehend aus Plastikteilen bestehendem Erscheinungsbild liegen mag. Aufgrund dieser Fahrzeugcharakteristiken - offenes und positives Erscheinungsbild sowie langsame Fahrgeschwindigkeit - ergibt sich, dass Unbeteiligte dem Fahrzeug nahezu einen persönlichen Charakter zuordnen, ähnlich wie das beispielsweise in Filmen wie „Nr. 5 lebt“, „Matrix“ oder „Krieg der Sterne“ der Fall ist. Auch dort ist zu beobachten, dass Robotern je nach Erscheinungsbild und Leistungsfähigkeit bzw. Aggressivität (ausgedrückt durch Fahrgeschwindigkeit) unterschiedliche Charaktere zugeordnet werden. Diese Eigenschaften und Mechanismen sollten vorsichtig in Betracht gezogen werden, wenn ein selbstfahrendes Fahrzeug in der Öffentlichkeit eingesetzt wird. Es scheint so zu sein, dass der Charakter eines Fahrzeugs ein wichtiger Bestandteil ist, um in der Öffentlichkeit positive Reaktionen zur Fahrzeugautomatisierung auszulösen, d.h. dass das automatisierte Fahrzeug durch entsprechende Mechanismen entweder als ,untergebener Diener“ oder „,rücksichtsloser Söldner" wahrgenommen wird (s. hierzu auch Kap. 3).

Aus den Begegnungen mit Passanten und Beobachtern ergeben sich immer wieder viele Fragen zu dem selbstfahrenden und individuell abrufbaren Personentransportsystem. Die häufigste Frage ist, wann und wo diese Fahrzeuge in der Öffentlichkeit nutzbar sein werden. Andere Fragen beziehen sich auf technische Spezifikationen der Fahrzeuge. Mitunter wird auch gefragt, ob und wie die Fahrzeuge auf Objekte reagieren, wobei ganz gezielt nach Fußgängern, Hunden, Katzen oder stationären Hindernissen gefragt wird. Gelegentlich ergeben sich auch Fragen zur Überwachung und Betriebshaftung der Fahrzeuge. Diese Fragen belegen allgemein das öffentliche Interesse an den Fahrzeugen und auch, dass die Öffentlichkeit sich viele Gedanken zum Betrieb sowie zu Beschränkungen von automatisierten Fahrzeugen macht. Das unterstreicht auch, dass eine Aufklärung und Information der Öffentlichkeit vor bzw. während des Betriebs dieser Fahrzeuge wichtig ist.

\subsection{Zusammenfassung und Ausblick}

Der Beitrag beschreibt die ersten Schritte zur Einführung eines selbstfahrenden und individuell abrufbaren Personentransportsystems. Bei dem Transportsystem handelt es sich um Fahrzeuge, die per Smartphone-App abrufbar sind und Personen innerhalb eines fest- 
gelegten Gebiets, wie beispielsweise eines Innenstadtbereichs, ohne direkte Eingriffsmöglichkeit und ohne Schienenführung befördern. Im hier beschriebenen Beispiel sollen diese Fahrzeuge an der Stanford Universität für wissenschaftliche Untersuchungen auf dem Gebiet innovativer Mobilitätslösungen mittels selbstfahrender Transportsysteme betrieben und dazu zunächst bezüglich ihrer Tauglichkeit für diese Arbeiten bewertet werden.

In den ersten Schritten wurden die Risikobewertung sowie Rechtseinordnung vorgenommen und dann das Betriebsgebiet sowie die Betriebsszenarien festgelegt. Dabei zeigte sich, dass zwar auf bestehende Regelungen und Vereinbarungen aufgebaut werden kann, das hier betrachtete Transportsystem durch den selbstfahrenden Charakter aber Anforderungen hat, die deutlich über das Bestehende hinausgehen. Dabei war es in allen Fällen von Vorteil, dass die Fahrzeuge langsam fahren (maximal $20 \mathrm{~km} / \mathrm{h}$ ) und ihr Einsatzgebiet begrenzt ist. Zusätzlich ist Betriebspersonal entweder direkt im oder am Fahrzeug oder per Funkverbindung mit dem Fahrzeug verbunden, was die Ersteinführung eines solchen Systems erleichtert.

Bei den notwendigen internen Koordinationsaufgaben sowie zu erstellenden Regelungen hat es sich als sehr wichtig erwiesen, den Betroffenen bzw. Entscheidern das Transportsystem im realen Einsatz vorzuführen, sodass das Betriebsrisiko sowie Möglichkeiten und Grenzen der Fahrzeuge realistisch eingeschätzt werden können. Das offene und positive Erscheinungsbild der Fahrzeuge gepaart mit großem Interesse sowie Neugierde in der Öffentlichkeit trägt dazu bei, dass die Fahrzeuge positive Reaktionen auslösen. Daran sind auch Erwartungen von Verbrauchern geknüpft, dass selbstfahrende Fahrzeuge in absehbarer Zeit vielen das oftmals lästige Autofahren in der Stadt abnehmen werden.

Die Unsicherheiten bei der Einführung des selbstfahrenden Personentransportsystems sind neben infrastrukturellen, betriebswirtschaftlichen sowie unternehmerischen Variablen durchaus auch die gesetzlichen Rahmenbedingungen. In dem hier betrachteten Beispiel der Einführung eines solchen Transportsystems an der Stanford Universität, die letztlich einen lokalen Rechtsbereich innerhalb des Bundesstaats Kalifornien darstellt, zeigt sich, dass der Betrieb auf Stanford-eigenem Gebiet vorteilhaft ist, da die Universität hier entsprechende Regelungen vorgeben kann. Allerdings wird derzeit für Kalifornien eine Gesetzesregelung für den Betrieb automatisierter Fahrzeuge verfasst, die dann für Durchgangsstraßen an der Universität auch rechtswirksam wäre. Damit wird in naher Zukunft zu sehen sein, wie die Rechtsprechung derartige selbstfahrende Fahrzeuge behandelt und wie der Betrieb bzw. die Ausführung des Transportsystems geartet sein muss.

Abschließend ergibt sich für den Ausblick zumindest aufgrund der hier gefundenen ersten Erfahrungen eine weitgehend positive Grundstimmung, als dass der Einführung selbstfahrender Transportsysteme in der Öffentlichkeit sehr wohlwollend gegenübergestanden bzw. diese gefördert wird. Es ist dabei allerdings anzumerken, dass diese ersten Eindrücke noch keine repräsentative Studie darstellen, sodass noch weitere Arbeiten zu Umsetzung und Akzeptanz selbstfahrender Fahrzeuge vorgeschlagen werden. Insgesamt ist damit aber zu hoffen, dass selbstfahrende und individuell abrufbare Personentransportsysteme langfristig einen deutlichen Beitrag zur Verbesserung der Individualmobilität in Städten leisten werden. 


\section{Literatur}

1. SAE International, "Taxonomy and Definitions for Terms Related to On-Road Motor Vehicle Automated Driving Systems" (16. Jan 2014)

2. Induct Navia Produktbeschreibung, Unternehmenswebseite, http://induct-technology.com/en/ products/navia-the-100-electric-automated-transport (abgerufen 28. Jun 2014)

3. "Induct presents world's first fully-electric driverless shuttle: the Navia", Induct Pressemitteilung, http:/induct-technology.com/en/files/2012/12/Navia-press-release.pdf (6. Dez 2012, abgerufen 28. Jun 2014)

4. "Le Cybus d'Inria en démonstration à la Rochelle", INRIA Pressemitteilung, http://www.inria. fr/content/download/9864/353310/version/1/file/Cybus-Inria.pdf (12. Mai 2011, abgerufen 28. Jan 2014)

5. Counts, N., "SMART Driverless golf cart provides a glimpse into a future of autonomous vehicles", MIT News, http://newsoffice.mit.edu/2013/smart-driverless-golf-cart-provides-a-glimpse-into-afuture-of-autonomous-vehicles (9. Dez 2013, abgerufen 28. Jun 2014)

6. "AKKA link\&go 2.0 electric self-driving concept designed for future cities", Designboom, http:// www.designboom.com/technology/akka-linkgo-2-0-electric-driverless-concept-car-for-the-cityof-the-future-03-12-2014/ (12. Mrz 2014, abgerufen 28. Jun 2014)

7. "Induct Launches Navia, The First 100 Percent Electric, Self-Driving Shuttle In The U.S.”, Induct Pressemitteilung, http://www.prnewswire.com/news-releases/induct-launches-navia-the-first100-percent-electric-self-driving-shuttle-in-the-us-238980311.html (6. Jan 2014, abgerufen 28. Jun 2014)

8. Zhang, R., Pavone, M., "Control of Robotic Mobility-On-Demand Systems: a QueueingTheoretical Perspective", Robotics: Science and Systems Conference (2014)

9. "DMV Adopts Autonomous Vehicle Testing Rules", California Department of Motor Vehicles (DMV) Pressemitteilung, http://www.dmv.ca.gov/pubs/newsrel/newsrel14/2014_34.htm (19. Mai 2014, abgerufen 28. Jun 2014) 